ORIGINAL ARTICLE

\title{
Eccentric decline squat protocol offers superior results at 12 months compared with traditional eccentric protocol for patellar tendinopathy in volleyball players
}

\author{
M A Young, J L Cook, C R Purdam, Z S Kiss, H Alfredson
}

Br J Sports Med 2005;39:102-105. doi: 10.1136/bjsm.2003.010587

See end of article for authors' affiliations

.....................

Correspondence to: Mark Young,

Musculoskeletal Research Centre, La Trobe

University, Bundoora,

Victoria 3086, Australia; mark.young@latrobe. edu.au

Accepted 4 February 2004
Background: Conservative treatment of patellar tendinopathy has been minimally investigated. Effective validated treatment protocols are required.

Objectives: To investigate the immediate (12 weeks) and long term (12 months) efficacy of two eccentric exercise programmes for the treatment of patellar tendinopathy.

Methods: This was a prospective randomised controlled trial of 17 elite volleyball players with clinically diagnosed and imaging confirmed patellar tendinopathy. Participants were randomly assigned to one of two treatment groups: a decline group and a step group. The decline group were required to perform single leg squats on a $25^{\circ}$ decline board, exercising into tendon pain and progressing their exercises with load. The step group performed single leg squats on a $10 \mathrm{~cm}$ step, exercising without tendon pain and progressing their exercises with speed then load. All participants completed a 12 week intervention programme during their preseason. Outcome measures used were the Victorian Institute of Sport Assessment (VISA) score for knee function and $100 \mathrm{~mm}$ visual analogue scale (VAS) for tendon pain with activity. Measures were taken throughout the intervention period and at 12 months.

Results: Both groups had improved significantly from baseline at 12 weeks and 12 months. Analysis of the likelihood of a 20 point improvement in VISA score at 12 months revealed a greater likelihood of clinical improvements in the decline group than the step group. VAS scores at 12 months did not differ between the groups.

Conclusions: Both exercise protocols improved pain and sporting function in volleyball players over 12 months. This study indicates that the decline squat protocol offers greater clinical gains during a rehabilitation programme for patellar tendinopathy in athletes who continue to train and play with pain.
$\mathrm{P}$

atellar tendinopathy (jumper's knee) occurs in several sports, with jumping athletes being the most susceptible. ${ }^{1}$ For example, the prevalence of jumper's knee is $40-$ $50 \%$ among elite volleyball players. ${ }^{2}{ }^{3}$ This often recurrent condition can severely limit or even end an athletic career, and recovery from each episode can be prolonged. ${ }^{4}$ Importantly, the condition is not self limiting, and, although rest may offer symptomatic relief, pain often recurs on resumption of activity. Surgical options have provided limited success, with a retrospective study finding that only half of all athletes who had either open or arthroscopic patellar tenotomy were competing at their former sporting level at a four year follow up. ${ }^{5}$

Tendinopathy research to date has concentrated on the morphology and aetiology of this condition, with little focus on conservative treatment options. Although testing the efficacy of clinical intervention protocols does not identify the underlying pathomechanics, it does provide invaluable clinical information on the usefulness of these protocols.

Conservative treatment of patellar tendinopathy is empirically and clinically based, with progressive eccentric strengthening forming the cornerstone of most rehabilitation programmes. Despite this, there is little clinically relevant research on which to base an eccentric training programme, as there are few randomised controlled trials that have compared exercise based protocols for this condition. ${ }^{67}$ Although speed based eccentric exercise programmes proposed by Curwin and Stanish ${ }^{8}$ are in widespread use, the efficacy of these programmes for patellar tendinopathy has not been fully investigated.
The success of a pain based eccentric exercise programme has been demonstrated in the treatment of Achilles tendino-

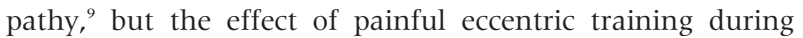
the rehabilitation of patellar tendinopathy is unknown. Furthermore, recent investigations have shown that squats performed on a $25^{\circ}$ decline board target the knee extensor mechanism more specifically than the standard squat, and it has been suggested that this may be relevant in the management of patellar tendinopathy. ${ }^{10}{ }^{11}$ Given these findings, a combination of squatting on a decline board and into tendon pain would theoretically provide an effective conservative treatment protocol for patellar tendinopathy.

The purpose of this study was to investigate the short and long term efficacy of two exercise programmes, one based on a traditional eccentric protocol (step group) and one based on more contemporary treatment options (decline group), for the treatment of patellar tendinopathy in volleyball players throughout a competitive season.

\section{METHODS}

This prospective randomised controlled trial used a parallel group, repeated measures design with an intervention period of 12 weeks and follow up at 12 months. Participants were recruited from the elite Victorian State League Volleyball (Australia) competition and clinically assessed (JC) on the last weekend of the regular season (2001). The La Trobe

Abbreviations: VAS, visual analogue scale; VISA, Victorian Institute of Sport Assessment 

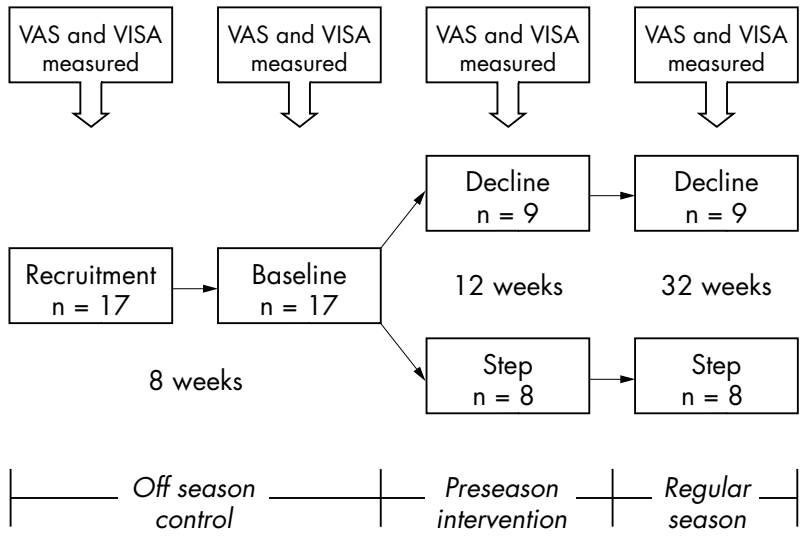

Figure 1 Progress of participants through the season and timing of interventions. VAS, visual analogue score; VISA, Victorian Institute of Sport Assessment.

University Human Ethics Committee approved the project, and all participants provided written informed consent.

Participants had to be aged 18-35 years and present with proximal patellar tendon pain that limited sporting function. To be included, they had to have tendon pain on palpation, symptom reproduction on jumping, squatting, and/or stepping, absence of referred pain outside the tendon, a Victorian Institute of Sport Assessment (VISA) score less than 80 points, and an abnormal (hypoechoic) ultrasound. Participants were excluded if they had undergone any knee surgery in the preceding 12 months, patellar tendon surgery, corticosteroid injection into the tendon, or anterior cruciate ligament reconstruction (patellar tendon), presented with patellofemoral pain, or were receiving adjunct treatment for knee pain. Participants with bilateral knee pain were asked to select the most symptomatic knee for the purposes of the study.

\section{Outcome measures}

The primary outcome measure used was the VISA score, designed specifically to quantify knee function in athletes with patellar tendinopathy..$^{12}$ Ranging from 0 to 100 , the VISA score consists of eight questions assessing symptoms and simple tests of function and ability to play sport. A maximum score of 100 points represents full pain free function. Competing athletes with patellar tendinopathy commonly record a score of 50-80 points. ${ }^{4}$ The $100 \mathrm{~mm}$ visual analogue scale (VAS) was used as a secondary outcome measure for
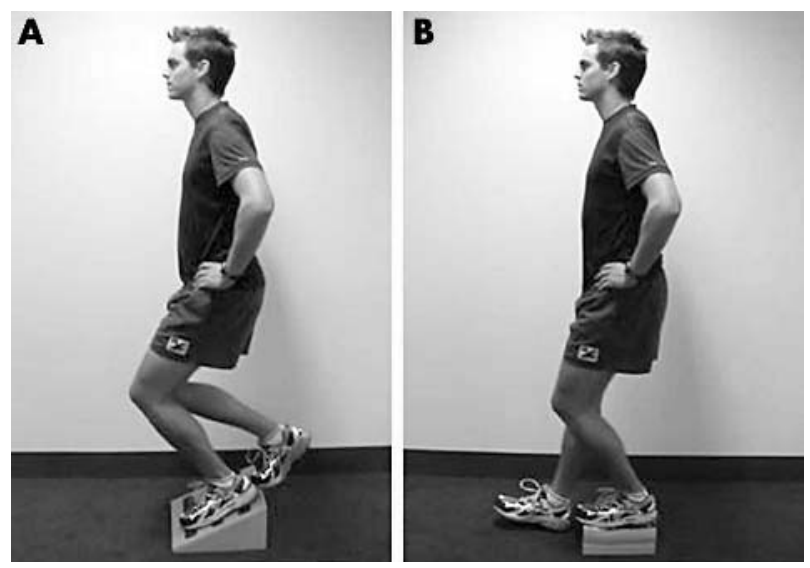

Figure 2 (A) Decline squat. (B) Step squat. average tendon pain during weekly activity. Both measures have been shown to be valid and reliable..$^{12-14}$

To assess the stability of initial scores, participants were assessed before and after an eight week control period between recruitment and baseline testing (fig 1). After baseline testing, participants assigned themselves to a group by randomly drawing from a batch of sealed, shuffled envelopes. VISA and VAS scores were recorded every four weeks during the intervention period and at 12 months. Investigators were blinded to all VAS and VISA measurements throughout the study. Participants were required to complete a daily training diary during the intervention period. This diary was used to monitor compliance, additional activity, and any drugs used.

\section{Intervention}

Participants were required to complete their exercises twice a day for 12 weeks. Both groups completed three sets of 15 repetitions during a session. All exercises were completed on a single (affected) leg to about $60^{\circ}$ of knee flexion, with participants being taught $60^{\circ}$ of knee flexion during their initial session. Both groups progressed load by adding weight to a backpack in $5 \mathrm{~kg}$ increments.

The decline group used a $25^{\circ}$ decline board to perform their squats (fig 2A). They completed the downward component (eccentric phase) of the squat on the symptomatic leg, and the upward component (concentric phase) on the asymptomatic leg. They were instructed to exercise into moderate tendon pain and to progress by increasing load if this pain eased. Participants in the step group used a $10 \mathrm{~cm}$ step to perform their squats (fig 2B). They completed both components of the squat on the symptomatic leg. They were instructed to exercise with minimal tendon pain only and progress their speed from slow to fast, as outlined in the
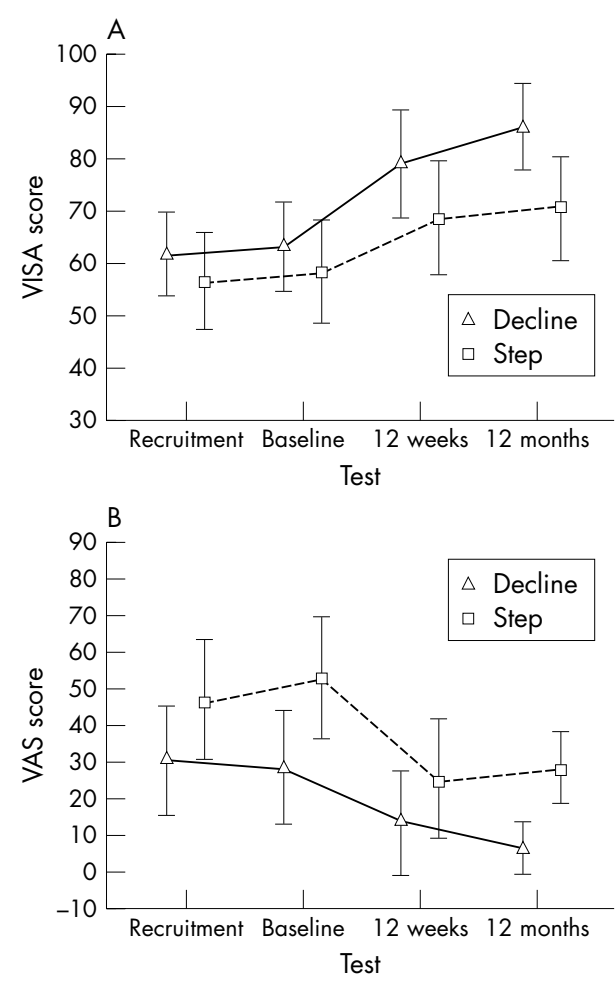

Figure 3 Mean (SD) change in (A) Victorian Institute of Sport Assessment (VISA) scores and (B) visual analogue scale (VAS) scores over 12 months. 

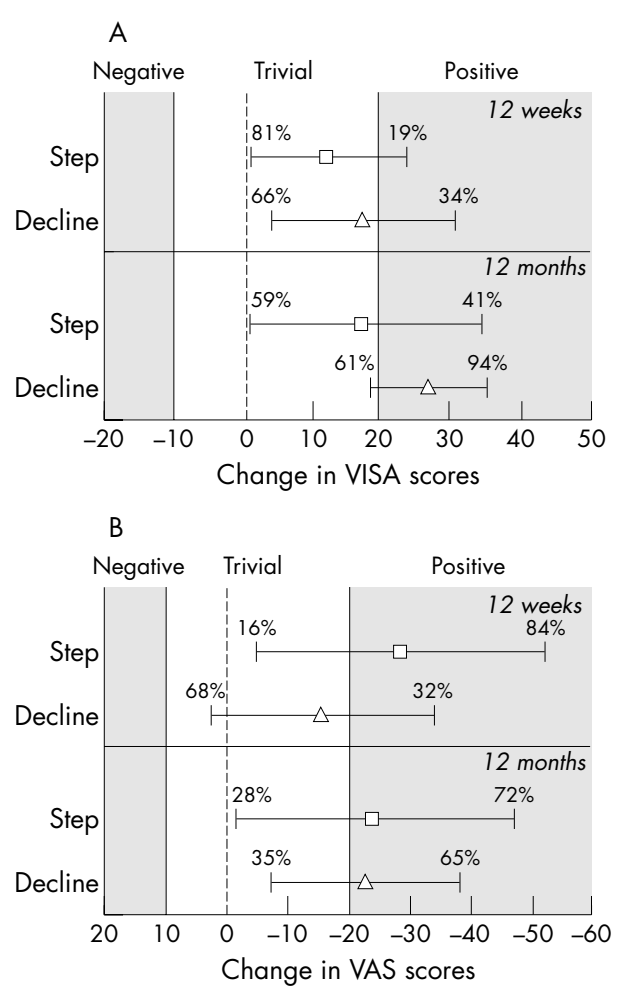

Figure 4 Likelihood of clinical outcomes for (A) Victorian Institute of Sport Assessment (VISA) scores and (B) visual analogue scale (VAS) scores at 12 weeks and 12 months (mean change $195 \%$ confidence interval)). VAS scores are inverted so that an improvement is signalled by a positive change.

Curwin and Stanish protocol. ${ }^{8}$ When participants could complete their squats at a fast speed, they increased load.

\section{Analysis}

Paired $t$ tests were used to assess baseline differences within groups. Between group VISA and VAS scores were compared using repeated measures two way analysis of variance for group and time interactions. Alpha significance was set at 0.05 .

A more contemporary analysis ${ }^{15}$ was used to determine the likelihood of obtaining a clinically worthwhile outcome. The smallest clinically important change in VISA and VAS scores was set at 20 points for both outcomes, as a 20 point improvement was considered to reflect a significant change in functional capacity. ${ }^{12}$ Clinical significance was calculated by considering the probabilities that the effect was clinically positive (greater than 20 points), trivial (20 to -10 points), or negative (less than -10 points) for each participant. To provide ease of interpretation, VAS scores were inverted for this analysis so that an improvement was signalled by a positive change, as with the VISA scores.

\section{RESULTS}

All participants ( 13 men, 4 women) completed the 12 week intervention and 12 month follow up (fig 1). There were no differences between the groups for subject age (mean (SD) 27.3 (1.8) years) or weight $(82.5(11.5) \mathrm{kg})$. There was a significant $(\mathrm{p}=0.01)$ difference in height between the groups (decline group $178.3(8.3) \mathrm{cm}$; step group 191.4 $(9.1) \mathrm{cm})$. This is probably because three women were randomised to the decline group and one to the step group. There was no difference between groups for exercise compliance, with a mean compliance of $72(18) \%$ of total possible sessions.
Statistical analysis revealed no change in outcome measures between or within groups over the eight week control period from recruitment to baseline testing (fig 3). Both groups had improved significantly $(\mathrm{p}<0.05)$ from baseline in both outcome measures at 12 weeks and 12 months, but there was no difference between groups for either outcome measure at any time.

Analysis of the likelihood of obtaining a clinical improvement in VISA scores at 12 weeks revealed no difference between groups. At 12 months, however, the decline group displayed a considerable difference in the likelihood of improvement in VISA scores (fig 4A). With the smallest practically important increase in VISA score set at 20 points, there was a $94 \%$ chance that the decline group would have a positive result at 12 months, compared with a $41 \%$ chance in the step group. Analysis of VAS score improvements revealed that the step group had an increased likelihood of reduced pain on VAS scores at 12 weeks compared with the decline group (fig 4B). There was no difference in improvement between the groups at 12 months.

\section{DISCUSSION}

Both the decline and step protocols were effective in the treatment of tendon pain and sporting function in athletes with patellar tendinopathy. Clinicians can confidently use both conservative protocols investigated in this study to positively affect pain and, more importantly, the ability to play sport in jumping athletes. However, over a 12 month period, the decline protocol gave a considerably greater likelihood of a 20 point or more improvement in VISA score.

A major strength of this study is that we recruited athletes who continued to compete with continuing tendon pain at an elite level during the previous season. Previous tendinopathy research has recruited participants who have disabling tendon pain, ${ }^{9}$ often to the extent that they have ceased or considerably reduced sports participation and are awaiting surgery. Athletes who continue to train and play with moderate tendon pain comprise a large contingent of the population who suffer from patellar tendinopathy. Thus the results of this study are particularly applicable to clinical sports medicine practice. After the preseason intervention, the athletes were then tracked for a full 12 month season with no further intervention. By following this group through an entire competitive season, we were able to examine the long term efficacy of the interventions within a more practical scenario rather than a more artificial, short term period, which has been a limitation of previous studies. ${ }^{67}$

Although a non-exercising control group was not used in this study, the use of an eight week control period assessed the stability of the outcome measures before the intervention began. The VISA and VAS scores taken at recruitment were compared with scores taken at baseline testing, with no intervention being administered during this control period. There was no significant change in outcome measures between or within groups over the eight week control period, and this supports clinical experience that suggests rest alone will not alleviate patellar tendinopathy pain.

The step group had a greater likelihood of reduced pain on VAS scores at 12 weeks than the decline group. This outcome was expected, as the decline group were training into tendon pain whereas the step group were avoiding tendon pain. An initial increase in tendon pain is expected during any pain based eccentric programme, ${ }^{9}$ and this possibility was explained to the participants at the beginning of the study. To ensure this expected increase in tendon pain did not interfere with the ability to maintain participation throughout the competitive season, all interventions were completed in the preseason period. There was no difference between groups in VAS scores for average tendon pain at 12 months, 


\section{What is already known on this topic}

There is little clinically relevant research on which to base a conservative rehabilitation programme for patellar tendinopathy.

\section{What this study adds}

Clinicians can confidently use the decline squat protocol investigated in this study to positively affect pain and, more importantly, the ability to play sport in jumping athletes.

and both groups completed the season with similar reductions in pain.

The purpose of this study was to compare contemporary treatment options (decline protocol) with a traditional eccentric programme (step protocol). Our ability to specify which component of the protocols was responsible for differences in improvement between treatment groups is limited because there was more than one variable between groups in this study design. Differences between groups were: squatting on a decline board or level ground, loading the eccentric phase only or both phases, exercising into tendon pain or short of pain, and progressing with speed then load or simply load. Comment on which aspect of the decline protocol was most effective in causing change in sporting function and tendon pain is reserved for more specific studies with greater numbers.

\section{CONCLUSIONS}

In active jumping athletes with patellar tendinopathy, both exercise protocols improved pain and sporting function. This study shows that the decline squat protocol presents a much greater chance of clinical improvement in VISA scores over a 12 month season than the step protocol. Therefore we recommend the use of the decline squat protocol during a rehabilitation programme for patellar tendinopathy in athletes who continue to train and play with pain. Further studies with larger numbers are required to confirm and further explore these findings.

\section{ACKNOWLEDGEMENTS}

We sincerely thank Peter Blanch, Dave Martin, Andrew Chapman, and Greg Lovell for their comments on this manuscript.

\section{Authors' affiliations}

M A Young, Musculoskeletal Research Centre, La Trobe University, Bundoora, Victoria, Australia, and Australian Institute of Sport, Bruce, ACT, Australia

J L Cook, Z S Kiss, Musculoskeletal Research Centre, La Trobe University C R Purdam, Australian Institute of Sport, Bruce, ACT, Australia H Alfredson, University of Umea, Umea, Sweden

Patient consent has been obtained for figure 2

Competing interests: none declared

\section{REFERENCES}

1 Ferretti A. Epidemiology of jumper's knee. Sports Med 1986;3:289-95.

2 Ferretti A, Papandrea P, Conteduca F. Knee injuries in volleyball. Sports Med 1990;10:132-8.

3 Lian O, Holen KJ, Engebretsen L, et al. Relationship between symptoms of jumper's knee and the ultrasound characteristics of the patellar tendon among high level male volleyball players. Scand J Med Sci Sports 1996;6:291-6.

4 Cook JL, Khan KM, Harcourt PR, et al. A cross sectional study of 100 athletes with jumper's knee managed conservatively and surgically. The Victorian Institute of Sport Tendon Study Group. BrJ Sports Med 1997;31:332-6.

5 Coleman BD, Khan KM, Kiss ZS, et al. Open and arthroscopic patellar tenotomy for chronic patellar tendinopathy. A retrospective outcome study. Victorian Institute of Sport Tendon Study Group. Am J Sports Med 2000;28: 183-90.

6 Cannell LU, Taunton JE, Clement DB, et al. A randomised clinical trial of the efficacy of drop squats or leg extension/leg curl exercises to treat clinically diagnosed jumper's knee in athletes: pilot study. Br J Sports Med $2001 ; 35: 60-4$.

7 Jensen K, Di Fabio RP. Evaluation of eccentric exercise in treatment of patellar tendinitis. Phys Ther 1989;69:211-16.

8 Curwin S, Stanish WD. Tendinitis: its etiology and treatment. Lexington: Collamore Press, 1984.

9 Alfredson $H$, Pietila $T$, Jonsson $P$, et al. Heavy-load eccentric calf muscle training for the treatment of chronic Achilles tendinosis. Am J Sports Med 1998;26:360-6.

10 Purdam CR, Johnsson P, Alfredson $\mathrm{H}$, et al. A pilot study of the eccentric decline squat in the management of painful chronic patellar tendinopathy Br J Sports Med 2004;38:395-7.

11 Purdam C, Cook J, Hopper D, et al. Discriminative ability of functional loading tests for adolescent jumper's knee. Physical Therapy in Sport 2003:4:3-9.

12 Visentini PJ, Khan KM, Cook JL, et al. The VISA score: an index of severity of symptoms in patients with jumper's knee (patellar tendinosis). Victorian Institute of Sport Tendon Study Group. J Sci Med Sport 1998;1:22-8.

13 Downie W, Leatham P, Rhind V, et al. Studies with pain rating scales. Ann Rheum Dis 1978:37:378-81.

14 Flandry F, Hunt J, Terry G, et al. Analysis of subjective knee complaints using visual analog scales. Am J Sports Med 1991;19:112-18.

15 Hopkins WG. A new view of statistics. In: Internet Society for Sport Science, 2000. www.sportsci.org/resource/stats.html (accessed 30 Nov 2001). 


\section{PostScript}

\section{BOOK REVIEWS}

\section{Keeping in time with your body clock}

Edited by J M Waterhouse, D S Minors, M E Waterhouse, T Reilly, and G Atkinson. London: Oxford University Press, 2002, £9.99, pp 199, softcover. ISBN 0198501748.

The book was difficult to access as it was very broad based and general and tended to be repetitive. It was difficult to identify the most suitable audience. Although it contained examples from research studies, no referencing to source material was included.

\section{Rating}

- Presentation

- Comprehensiveness

- Readability

- Relevance

$10 / 20$

- Evidence basis

$10 / 20$

- Total

$8 / 20$

$43 / 100$

M O'Brien

\section{The practical guide to range of motion assessment}

Edited by J Gerhardt, L Cocchiarella, D Randall. Published by American Medical Association Press USA, 2002, US $\$ 69.95$, hardcover, pp 108. ISBN 157947263X

This text has been written as additional instruction to the American Medical Association guides to the evaluation of permanent impairment (5th edition, 2000), and Master the Guides (5th edition, 2001). Primarily for physicians, this text is also relevant to other healthcare professionals. The authors, who have a long standing association with issues relating to measurement of joint range of motion, aim to achieve greater accuracy and reliability of measurement by the use of standardised protocols, instrumentation, and reporting. This is a laudable goal as the patient's range of motion and the expected normal range of motion are used with other measures as a basis for evaluation of percentage permanent impairment (American Medical Association, 1993). In countries adopting these impairment ratings, such measurements form a substantial component of the permanent disability compensation award, which translates directly into the dollar amount paid to the patient by a compensatory body.

The book is divided into two main sections. Part 1 describes principles for measurement of joint range of motion. Part 2 provides instructions for range of motion measurement of individual joints and spinal regions.

In Part 1 , sound recommendations are made for preparing the subject, both physically and psychologically. Information on factors and conditions that may be associated with altered motion are tabulated, and a list of possible warm up exercises included. Instructions are also provided to facilitate reliable identification of relevant spinal landmarks such as the spinous processes of $\mathrm{Tl}$, $\mathrm{T} 12$, and S2.

A comparison is made of the two instruments mainly used for measurement of joint range of motion: the two arm (universal) goniometer and the gravity based inclinometer. However, despite the authors' preference for the inclinometer, the two measurement instruments appear to share sources of error more closely than suggested. In this respect, although the "step by step" pictures aim to facilitate understanding of the measurement technique, the examiner sometimes appears to have been photographed to maximise the view of the inclinometer location rather than the optimal examiner position. It can be appreciated that because of the possible sources of error outlined, the difference between a valid measure and an exercise in futility may be small with either instrument.

The SFTR system of recording developed by these authors is well described in the text and illustrated in accompanying tables. There may be some confusion for readers where currently accepted conventions for naming movements have been changed "for practical purposes"-for example, "upper ankle" joint (hind foot) plantar and dorsiflexion, and "lower ankle" joint (hind foot) inversion and eversion. As the movements of the foot are complex, and often misunderstood, an approach consistent with the current literature should preferably have been maintained.

Part 2 of this book provides descriptions and clear photographic illustrations of techniques for the measurement of the range of spinal and peripheral joint motion. One of the key principles in measurement of range of joint motion is to exclude, wherever possible, movement of adjacent joints, often termed "trick movement".

Thus it is pleasing to see in this text that spinal motion is measured using two inclinometers and tangents to the curve over the limit vertebra of the curve of interest.

Relevant landmarks for each anatomical segment are listed at the top of each page. However, it is not always easy to determine which of the landmarks in the series is pertinent to a particular movement. For those less familiar with measurement these landmarks may be better described for each individual joint motion

Aiming for reproducibility, the authors recommend establishing a neutral or zero starting position of the joint with reference to gravity. Although this recording method clearly allows a variety of possible movement limitations, no suggestions are provided for adapting the method when the subject cannot adopt the neutral position-for example, in the wrist after Colles fracture or in cases of knee flexion contracture. In addition, for the measurement of some lower limb joints, the method described is not entirely practical. For example, during hip extension, all but the most athletic may have difficulty in adopting the start position.

Another disadvantage of a standardised technique is that errors may occur if the examiner fails to fully comprehend the underlying principles. For example, the examiner may need to be made aware that the accuracy of measurement from a single inclinometer relies on the adjacent body segment (usually proximal) being either vertical or horizontal and remaining stationary during the movement. Thus for the new examiner, some instructions in the text for preventing likely "trick" movements during the test may be beneficial.

References reporting the reliability of "inclinometry" have been provided. However, as a "stand alone" text on measurement of joint motion, it is disappointing that the results of research supporting the measurement techniques described have not been included

The goal of obtaining reliable and valid measurement of joint range of motion continues to present a challenge to the clinician and researcher alike. Multiple possible sources of error remain to be overcome. Overall, the authors have attempted to minimise measurement variability by providing standardised measurement processes. Unfortunately improved reproducibility may not always guarantee the validity of the measurement, which is relevant when the patient's range of motion is compared with normative data for evaluation of impairment. It is recognised that it is difficult to develop a standardised measurement technique that adequately addresses all issues and can be applied to all joints and regions. Nevertheless, this book represents another step in the right direction.

\section{Analysis}

- Presentation

- Comprehensiveness $16 / 20$

- Readability

- Relevance

- Evidence basis

$15 / 20$

- Total

$15 / 20$

$16 / 20$

$10 / 20$

$72 / 100$

\section{Science and soccer, 2nd edition}

Edited by T Reilly, A M Williams. London: Published by Routledge, 2003, £24.99, softcover, pp 332. ISBN 0415262321

The editors are to be congratulated for their work in the field over the years represented in this text which has arisen from a series of World Congresses of Science and Football which began in 1987 in Liverpool. Unfortunately this also reveals a weakness in the limited pool of authors from which the text draws, the majority of whom list their professional addresses as Liverpool. As an Aussie with an AFL background, it may be a bit presumptuous of me to criticise those from the birthplace of the game (and the current home of a champion Australian player). However, bearing in mind recent international results, it is fair to assume that some sports science knowledge in soccer may 
exist in places such as South America, Asia, Africa, and possibly Greece! And what was the score at Upton Park in 2003 anyway!

This text is certainly comprehensive in the areas it covers and easily read from the first whistle to the full 90 minutes. As stated, it covers every key facet of the game including players' anatomy, physiology, biomechanics and psychology, coaching and training methods, nutrition, injury prevention and rehabilitation, surfaces and equipment, match analysis, growth and development of younger players, and talent identification. The allocation of space given to each area perhaps fairly reflects the strengths and areas of interest of the contributors. If readers of this journal such as team doctors and physiotherapists are after detail on sports medicine and injuries in soccer, they will be disappointed particularly by the 10 page chapter on injury prevention and rehabilitation.

The value for many readers in this text perhaps lies in the knowledge and understanding they can gain in fields of the sports science professionals around them, rather than their own fields. An understanding of the psychology of injury, coaching, and skill acquisition is provided in some detail and will doubtless improve communication and understanding between team support staff. Other chapters such as those on nutrition and environmental issues provide excellent practical advice for team physicians planning team travel and season routines. I am not sure how the chapter on soccer hooliganism fits into the rest of its section covering coaching science-perhaps this is better in a sociological text.

As a medical professional actively working in soccer, I certainly found this text a useful read and it will retain a place on my bookshelf. It certainly improves my knowledge in the fields of the sports scientists who work with our teams and thus helps me converse from a position of understanding. And of course it will be of value to those undertaking the masters course in Liverpool. For a reference text for sports injuries and soccer, I suggest you look elsewhere.

\section{Rating}

- Presentation $13 / 20$

- Comprehensiveness

- Readability

- Relevance

- Evidence basis

- Total

$13 / 20$

$17 / 20$

$15 / 20$

$17 / 20$

$75 / 100$

(PS: The score was Australia 3, England 1!)

A Jowett

\section{CALENDER OF EVENTS}

\section{UK Radiological Congress 2005} (UKRC 2005)

6-8 June 2005, Manchester, UK

The UK Radiological Congress (UKRC) meeting will encompass the medical, scientific, educational, and management issues that are of interest and relevance to all those involved in the diverse fields of radiological sciences and oncology.

The UKRC provides a forum in which to bring together clinicians, scientists, radiographers, technicians, and other professionals to present and discuss the latest developments and challenges in diagnostic imaging, radiotherapy, and allied radiological sciences.

Key subjects to be covered include: diagnostic radiology; ultrasound; nuclear medicine; interventional radiology; veterinary radiology; emerging technologies; image analysis; computer applications; PACS; radiobiology; radiological physics; management \& audit; computed tomography; magnetic resonance; equipment development.

Expected attendance (conference and exhibition): 4000

Further details: UKRC 2005 Organisers, PO Box 2895, London WlA 5RS, UK; Website: www.ukrc.org.uk; Fax: +44 (0)20 7307 1414; Conference tel: +44 (0)20 7307 1410, Email: conference@ukrc.org.uk; Exhibition tel: +44 (0)207307 1420, Email: exhibition@ukrc. org.uk

\section{1 st World Congress on Sports Injury Prevention}

23-25 June 2005, Oslo, Norway

This congress will provide the world's leading sports medicine experts with an opportunity to present their work to an international audience made up of physicians, therapists, scientists, and coaches. The congress will present scientific information on sports injury epidemiology, risk factors, injury mechanisms and injury prevention methods with a multidisciplinary perspective. Panel discussions will conclude symposia in key areas providing recommendations to address the prevention issue in relation to particular injuries and sports.

Further details: Oslo Sports Trauma Research Centre and Department of Sports Medicine, University of Sport and Physical Education, Sognsveien 220, 0806 Oslo, Norway._Email: 2005congress@nih.no; website: www.ostrc.no

\section{Osteosynthese International 2005}

15-17 September 2005, Curiohaus, Hamburg Congress-Chairman: Johannes M. Rueger, M.D., Professor and Chair

Topics:
- Innovations in intramedullary osteosynthesis

- New frontiers in osteoporosis and fracture treatment

- Current trauma research

- Special topic: Recent development in pelvic and acetabular fractures

Abstract submission deadline: 31 March 2005

Further details: INTERCONGRESS GmbH, Martin Berndt, Düsseldorfer Str. 101, 40545 Düsseldorf-Germany. Tel: +49 $211585897-$ 80; fax: +49 211 585897-99; email: martin. berndt@intercongress.de; website: www. osteoint2005.de

\section{4th European Sports Medicine Congress}

13-15 October 2005, Lemesos, Cyprus

Further details: Email: pyrgos.com@ cytanet.com.cy

\section{BASEM Conference 2005}

10-12 November 2005, Edinburgh, Scotland Further details: Email: BASEMinfo@ aol.com

BASEM Conference 2006

5-7 October 2006, Oxford, UK

Further details: Email: BASEMinfo@ aol.com

\section{CORRECTIONS}

doi: 10.1136/bjsm.2003.010587corrl

Young M A, Cook J L, Purdam C R, et al. Eccentric decline squat protocol offers superior results at 12 months compared with traditional eccentric protocol for patellar tendinopathy in volleyball players $(B r J$ Sports Med 2005;39:102-5). The total percentage scores in the final line of figure $4 \mathrm{~A}$ are incorrect; the correct totals are $6 \%$ and $94 \%$. We apologise for this error.

doi: 10.1136/bjsm.2003.009969corrl

Kordi R, Dennick R G, Scammell B E. Developing learning outcomes for an ideal MSc course in sports and exercise medicine (Br J Sports Med 2005;39:20-3). In table 2 of this paper the Queen Mary University of London course has been listed as an MSc in sports medicine. The course actually concerns sport and exercise medicine, and also offers diploma and certificate level qualifications. We apologise for this error. 